\title{
RÉFLEXions MéthodologiQues SuR L'Autoconfrontation Croisée
}

\author{
(Reflexões Metodológicas sobre a Autoconfrontação Cruzada)
}

\section{Daniel FAïTA * et Marcos VIEIRA **}

\begin{abstract}
We have studied and developed a methodological procedure, named cruised auto confrontation, in order to analyze work situations, with the purpose of recovering the process of production, through the understanding of the activity purpose itself. The principles of the Bakbtinian dialogism and the Vigotskyan development psychology were applied to understand linguistic interventions in professional activities. The five subsequent parts of the method belp the analyst orientate his work in order to develop an action towards the subject, which is confronted with himself and with others in the activity. The method succeeded in shedding light on the relation between real activity and represented activity, taking into account speech genders and activity gender.
\end{abstract}

KEY-WORDS: Cruised auto confrontation; Activity gender; Speech gender; Methodology.

RESumo: Estudamos e desenvolvemos uma metodologia de análise de situações de trabalho chamada autoconfrontação cruzada, buscando compreender o processo de produção do sentido na atividade. Mobilizamos o principio bakbtiniano do dialogismo e vigotskiano do desenvolvimento psicológico para compreender a intervenções de lingüistas em atividades profissionais. As cinco fases do método ajudam o analista a guiar o próprio trabalho e possibilitam o desenvolvimento do poder de agir do sujeito confrontado à sua atividade com os outros. A autoconfrontação expõe as relações entre o real vivido e a representação da atividade apontando para as relações entre gênero da atividade e do discurso.

PaLAVRAS-CHaVE: Autoconfrontação cruzada; Gênero da atividade; Gênero do discurso; Metodologia.

\footnotetext{
* Daniel FAÏTA est Directeur de Recherche à l'IUFM d'Aix-Marseille, chercheur au laboratoire d'Ergologie APST (Université de Provence), Associé à l'équipe de Clinique de l'Activité (CNAM, Paris). (DF@wanadoo.fr)

** Marcos VIEIRA est Psychiatre et Linguiste, professeur adjointe à la Faculté de Sciences Medicales à l'Université Fédérale du Mato Grosso (Brésil) et chercheur de la Fondation CAPES. (mvieir@hotmail.com).
}

D.E.L.T.A., 19:1, 2003 (123-154) 
Cet article tente de poser quelques problèmes méthodologiques relatifs à la pratique, désormais assez diffusée de la procédure d'autoconfrontation croisée (Clot et Faïta, ainsi que Clot, Faïta, Fernandez, Scheller). On cherchera avant tout à préciser l'apport du point de vue de l'analyse du rapport dialogique sur cette méthode. Les notions de dialogue, rapport dialogique, dialogisme, utilisées ici trouvent leur origine dans les travaux du " cercle de Bakhtine »(Bakhtine, 1977), et ont fait l'objet de débats, exégèses et développements importants (Todorov, 1981). Elles conduisent à prendre l'énoncé concret pour base matérielle de l'analyse des situations, des actes et des pensées humains, ce qui a pour conséquence de fournir aux sciences humaines un objet - le rapport dialogique - situé à la frontière entre discours et activité. Accepter cette position de principe conduit à rompre avec l'attitude consistant à isoler une partie de ce même objet, en privilégiant l'une de ses caractéristiques, à l'isoler et à la traiter sous l'angle d'une logique disciplinaire (philosophie, linguistique, psychologie, psychiatrie...).

\section{Clinique de l'activité et constitution du groupe d'analyse du travail dans un cadre dialogique.}

La méthode en clinique de l'activité ${ }^{1}$ propose donc de nouvelles modalités en analyse du travail, où le dialogisme constitue le principe directeur et la source du dispositif méthodologique. Il s'agit, pour l'essentiel, de créer un cadre où les partenaires associés à la recherche, les acteurs concernés, puissent à la fois peser sur les attendus et les conditions de cette recherche, et mettre en chantier des manières de penser collectivement leur travail, entre l'image filmée de ce qu'ils ont fait et la mise en discours de ce qu'ils sont en train de faire. Cette démarche institue ce que l'on a appelé un espace-temps où les acteurs ont la possibilité de mobiliser, où de rétablir leur « pouvoir d'agir » en différé par rapport aux actions exposées par le film.

\footnotetext{
1 Ces termes désignent en l'occurrence une démarche caractérisée dans la pratique par des options scientifiques et méthodologiques visant à favoriser un processus de développement des situations ainsi que du pouvoir d'agir des individus et des collectifs de travail, ainsi que l'équipe de recherche dirigée par Y.Clot dans le cadre du Laboratoire de Psychologie du Travail (CNAM, Paris).
} 
La méthode se structure généralement en trois phases: I. La constitution d'un groupe d'analyse (voir Faïta, 1997) et sa mise au travail sur l'objet de la recherche et les options méthodologiques. II. La réalisation des autoconfrontations simples et croisée et la conjugaison des expériences des participants. III. L'extension du travail d'analyse par la restitution de son produit au collectif professionnel.

Le présent article mettra l'accent sur les relations dialogiques structurant l'ensemble des trois phases, tout en se concentrant sur la constitution et le fonctionnement du groupe d'analyse, sur le rôle du chercheur-analyste, et sur la nature et la portée des résultats obtenus au terme des différents stades. Il s'agit selon nous d'un choix stratégique, dont l'effet recherché sera de consolider la base méthodologique du processus d'analyse du travail engagé selon des principes que nous référons à la théorie de l'activité dialogique due à Bakhtine, ainsi qu'aux hypothèses de Vygotsky sur le développement..

\section{Constitution du groupe d'analyse.}

La première phase, celle de la constitution du groupe, représentatif du " milieu de travail associé à la recherche »(Faïta, 2001a, p.274), nécessite un « long travail d'observation des situations et des milieux professionnels afin d'en produire des conceptions partagées avec les travailleurs » (Clot, Faïta, et al., p.21). Les « impasses » inévitables (Faïta, ibidem, p.272), consécutives aux erreurs de perspective et d'appréciation commises par le chercheur dans son apprentissage de la réalité du travail et des modalités de celui-ci, comme les erreurs commise par le collectif dans son appropriation des objectifs et contraintes de la recherche, constituent en fait la condition incontournable d'une bonne élaboration de ces conceptions. Un problème particulier se pose toutefois au cours de cette phase initiale, dont le traitement conditionne la validité du dispositif. Si en effet la participation plénière du collectif à la définition des modalités initiales du travail de co-analyse, comme, par exemple, le choix en son sein des acteurs participant aux phases ultérieures, ne soulève pas de problème majeur, la question du choix des séquences d'activité filmées soumises à l'autoconfrontation parait beaucoup plus épineuse.

On a pu voir (Faïta, Clot, et al., 1996, Faïta, 2001), que l'élection d'une séquence de conduite des trains par le collectif des agents de conduite 
pouvait aboutir à un processus très productif, mais il convient de ne pas oublier que ce choix intervenait après une longue période d'échange et de dialogue avec l'équipe de recherche, voire de controverses sur la manière de définir l'objet de celle-ci. Rappelons en effet cette expérience riche en enseignements : associé dès le début à la définition des objectifs, le collectif d'agents avait contesté les procédures d'investigation utilisées (observation en situation de travail, formulation d'hypothèses, mises à l'épreuve de cellesci devant le collectif, et production de conclusions). Ce même collectif avait alors revendiqué l'initiative, réunissant un petit groupe de cinq conducteurs avec pour mission d'élaborer et rédiger ensemble une analyse de ce qui était censé faire toute la complexité de leur activité. Le résultat en fut particulièrement décevant : quatre pages de rappels sommaires des procédures prescrites à mettre en œuvre, agrémentées de quelques commentaires anodins. Cela témoignait de l'attraction exercée par les modèles discursifs antécédents sur le vouloir-dire collectif (ou dessein discursif, Bakhtine, 1984: 286-287), phénomène passible de plusieurs explications. Il est certain que l'expression collective qui puise ses ressources dans le genre professionnel (Clot et Faïta, 2000), mémoire du milieu de travail et somme d'instructions disponibles pour les comment faire requis par les objectifs de l'action, tout comme le passage à l'écrit, ajoute un filtre supplémentaire à l'énonciation. Il est indiscutable, en définitive, qu'une accumulation de lois et contraintes, régissant l'énonciation, s'impose sans contre-parties à un collectif de travailleurs dépourvu des ressources critiques nécessaires pour discriminer les éléments pertinents et ceux qui ne le sont pas en matière de formalisation et représentation de leur propre activité sous la forme classique de la mise en mots.

L'examen d'une autre expérience, empruntée aux observations et débats préalables au choix d'une séquence d'activité en situation de consultation entre infectologistes et patients atteints du SIDA (Vieira, 2001), nous a montré que le collectif de médecins excluait la confrontation de leurs observations avec l'examen physique, arguant de l'absence d'intérêt de celui-ci dans le cas de ces patients. Cependant, les observations de l'analyste montraient l'existence de difficultés révélées par deux indices : l'attitude tendue des patients pendant la mise en ouvre des techniques d'examen, et leur absence de réponse aux questions visant à clarifier leurs sensations corporelles, posées par les infectologistes. Le contraste entre le « vouloirdire » de ces derniers et ces observations conduisit à retenir l'examen physique comme séquence à enregistrer. 
Il incombe donc au chercheur, partiellement, parfois totalement, d'envisager le choix de moments et séquences de l'activité en fonction de cette activité - plus exactement de la perception qu'il en a - plus que de la représentation discursive que le collectif est disposé à en fournir ${ }^{2}$. Il est plus que probable, en effet, que la représentation collective de l'activité repose au moins autant sur la façon dont chacun est en mesure de penser cette activité que sur ce que le locuteur collectif est disposé à dire d'elle, captant ou annihilant les velléités individuelles, ce qui a pour effet de déplacer le centre de gravité du débat. L'activité, en fin de compte, demeure à l'arrière-plan de ce processus et des compromis qui peuvent contribuer à l'occulter en partie. Il incombe donc à l'analyste de faire la part de ce qui, dans les options du collectif, relève d'un projet discursif spécifique, de manière à assurer la meilleure part à la représentation filmique des dimensions pertinentes de l'activité de travail.

L'expérience, en matière de clinique de l'activité, permet sur ces points de disposer de garanties appréciables (voir Faïta, 1997, et Clot et Faïta, 2000):

I. L'observateur ne renonce pas à défendre le point de vue de la recherche face aux choix argumentés par les acteurs;

II. L'action même de filmer privilégie la variété des séquences recueillies au cours d'une même journée de travail.

III. Les protagonistes de l'activité sont filmés dans des situations de travail aussi proches que possible les unes des autres, ce qui est essentiel pour engager l'autoconfrontation sur les manières de faire.

Ces remarques motivent un approfondissement des questions posées, et notamment du danger encouru si l'on ne prend pas suffisamment en considération les rapports entretenus entre l'activité initiale, comme discours et texte, et l'activité discursive en cours d'autoconfrontation, dans le champ de la pratique en analyse clinique de l'activité.

2 On s'expliquera plus loin sur ce qui fait considérer ici qu'une représentation filmique participe de la contrepartie discursive de l'activité. 


\section{Rôle de l'analyste dans la construction des matériaux de l'autoconfrontation.}

Pour éviter de procéder à une autoconfrontation à partir d'un matériau fondé sur le projet discursif des protagonistes, plus que sur une représentation de l'activité de ceux-ci, il est donc essentiel de ne rien abandonner au moment du choix des éléments constitutifs de ce matériau. Cela suppose que l'analyste n'oublie pas son rôle, participe à la sélection des supports techniques (images mais aussi discours enregistrés) de telle sorte que l'activité constitue bien l'objet construit sur la base de l'observation et des apprentissages antérieurs qu'il a consentis. Il ne doit à aucun moment confondre son rôle avec celui des acteurs, qui pour leur part pensent ce que pourrait ou devrait être leur travail en même temps qu'ils mettent en mots ce qu'ils en revoient. L'activité sur l'activité en quoi consiste l'autoconfrontation ne doit pas se substituer à l'activité elle-même, car à ce stade, le processus de production de sens, s'il se réalise à partir du travail observé, n'en demeure pas moins extérieur à lui, et surtout anticipe son déroulement, sous l'effet de contextualisation exercé par l'autoconfrontation simple. Il convient d'ailleurs, à ce propos, de distinguer deux modes de contextualisation successifs et articulés :

- l'autoconfrontation simple est le moment de la production d'un discours en référence aux séquences filmées, à ce qu'elles donnent à voir, suggèrent ou évoquent (ce que nous qualifions de " première source de signification concrète » dans le tableau ci-dessous).

- L'autoconfrontation croisée est l'espace-temps, en d'autres termes le moment de suspension au cours duquel la référence se globalise, où tout ce qui était dans l'informulé peut trouver à se révéler, parallèlement au processus déclenché par la procédure... Outre le film de l'activité initiale, en effet, avec ses lacunes et ses ellipses, l'autoconfrontation simple ajoute un contexte lesté de commentaires, de digressions métacognitives et métalinguistiques, d'anticipations sur le déroulement du procès de travail observé.

- La compréhension des mécanismes mis en œuvre repose donc sur la capacité de discerner en permanence les sources des discours, leur transformation, puis le développement global. 
On retrouve là l'essentiel de la conception bakhtinienne de la compréhension en tant que processus actif, selon laquelle comprendre c'est d'abord agir de telle sorte que l'on anticipe l'activité de l'autre en réponse à la sienne propre. C'est probablement inévitable, mais il convient de ne pas perdre de vue que l'objectif de l'analyse à suivre demeure précisément la mise en rapport proprement dialogique entre la situation représentée et le processus déclenché à partir d'elle. Les mouvements discursifs occasionnés chez les protagonistes par le film participent de la remise en chantier de ce qu'ils se voient faire et s'entendent dire, qui constituent autant d'éléments de décontextualisation-recontextualisation de leur activité actuelle. C'est seulement ainsi que l'on pourra établir des relations de pertinence entre le déjà vu, le déjà dit et vécu, et les phénomènes émergents. On pourra par conséquent ultérieurement donner sens à ceux-ci; c'est à dire relire le texte produit par chacun des protagonistes en se demandant comment les mots, structures, phénomènes divers, s'éclairent les uns les autres à distance plus ou moins grande:

\footnotetext{
"Derrière tout texte on trouve le système de la langue, ce qui y correspond, c'est tout ce qui est répétitif et reproductible, tout ce qui peut être donné hors du texte. Mais dans le même temps tout texte (en sa qualité d'énoncé) est individuel, unique et non reproductible, et c'est là que réside son sens (son dessein, ce pourquoi il a été crée). C'est par là qu'il renvoie au vrai, au véridique, au bien, au beau... (...) Au regard de cette fonction là, tout le répétitif et le reproductible sont de l'ordre du moyen, du matériau. Le texte c'est ce qui n'entre pas dans le cadre linguistique et philologique. Ce second aspect (pôle) est la propriété du texte lui-même mais ne se révèle qu'en situation et dans la chaîne des textes (dans l'échange verbal à l'intérieur d'un domaine donné). Ce pôle le relie non pas aux éléments reproductibles d'un système de langue (de signes) mais aux autres textes (non reproductibles) en un rapport particulier, dialogique (et dialectique si l'on fait abstraction de l'auteur)". (Bakhtine, 1984: 313-314).
}

Au moment crucial de l'autoconfrontation, l'analyste-participant doit donc centrer en permanence le débat sur l'activité. L'une des plus sûres garanties de la méthode réside donc, on l'a dit, dans sa capacité à maintenir le mouvement dialogique autour de ce que les protagonistes voient qu'ils font Il ne s'agit pas de réduire, d'édulcorer leur expression, mais au contraire de saisir avec un maximum de chances de succès les dimensions composites des significations concrètes, faites de l'association des énoncés produits et de ce que révèle leur relation effective à ce à quoi ils réferent. On ajoutera qu'une utilisation des pièces de cette première phase (film, 
autoconfrontation simple) privilégiant le dessein discursif des protagonistes ${ }^{3}$ au détriment de la remise en travail des éléments de l'activité fait courir le risque d'un échec final par l'appauvrissement, voire la neutralisation de l'effet retour exercé par la procédure au moment de la restitution au milieu de travail. Pour la même raison, la prolifération d'autoconfrontations croisées détachées du processus continu (autoconfrontations simples initiales, suivies des autoconfrontations croisées symétriques, précédant elles-même la diffusion consécutive des acquis sous la forme d'un retour vers le collectif), présente un danger aigu d'instrumentalisation et de perte de pertinence. Il nous semble justifié de transposer à cette problématique le postulat de Bakhtine suivant lequel « L'acte humain est un texte potentiel et ne peut pas être compris (en qualité d'acte humain, distinct de l'action physique) hors du contexte dialogique de son temps (dans lequel il figure au titre de réplique, de position de sens ...) »(Bakhtine, 1984:.316).

Les autoconfrontations simples représentent déjà un mode de réalisation et de diffusion des projets discursifs, ou simplement d'appropriation de la phase d'observation avant son retour sur le collectif. On doit rester vigilant face au danger d'instrumentalisation de ce premier support, repris et développé pendant l'autoconfrontation croisée, car le risque existe que le vouloir dire des protagonistes se substitue déjà à leur activité. Cela engagerait des conséquences néfastes pour l'analyse de l'activité de travail réelle, et donc pour le rétablissement du pouvoir transformateur de l'action collective.

La base de la démarche doit en effet demeurer la confrontation terme à terme des « comment faire » respectifs des acteurs, dessinés sur le fond des « à faire » et " pour faire », prescriptions et modes opératoires contenus par la tâche et l'activité qui s'y réalise concrètement. Le rôle du contexte supporté par le film, puis les développements parallèles occasionnés par la première autoconfrontation, sont déterminants pour la productivité d'une autoconfrontation croisée qui ne soit pas principalement l'occasion de productions discursives axées sur la conquête et l'illustration des places des interlocuteurs.

\footnotetext{
3 Cette remarque ne doit pas être considérée comme l'indice d'une exclusive prononcée à l'encontre de certains genres et procédés discursifs: il est parfaitement possible de mettre en mots une expérience professionnelle sous la forme du récit, ou encore en ironisant ou dramatisant la référence aux situations.
} 
Une approche clinique doit reposer sur la base primordiale du développement de la situation d'origine par l'autoconfrontation première, pour ensuite déployer le rapport dialogique par le développement de l'objet constitué, et ensuite des sujets dans le cadre de l'autoconfrontation croisée, au sens où le dialogue, par le rôle médiateur qu'y joue le langage, provoque et supporte à la fois la réalisation et la diversification des façons de penser le réel et les rapports entretenus avec ses éléments (Vygotsky, 1934/ 1985). C'est dans cette progression en effet que l'on a toute chance de toucher aux dialogues entre activité et activité sur l'activité, particulièrement l'activité verbale, discursive.

\section{Des thèses de Bakhtine vers l'autoconfrontation.}

Chez Bakhtine, la question du développement induit par le rapport dialogique est posée de manière intrinsèque par la distinction (et le lien, en même temps) entre genres de discours primaire et secondaire. Les premiers, en effet sont indissolublement confondus avec l'activité, car ils donnent leur substance aux échanges verbaux en même temps qu'ils guident leur déroulement au prix des changements, des ruptures (et/ou enchaînements) auxquels procèdent les interlocuteurs. Les seconds, avec leur vocation à la généralité, à la prise de distance avec la réalité concrète, supportent les différents processus d'abstraction, de conceptualisation, de métadiscursivité, etc. Ils sont néanmoins l'émanation des premiers dans le fonctionnement et la circulation desquels ils puisent leurs traits, comme le roman tire sa substance de la multitude des échanges quotidiens (Bakhtine, Esthétique et théorie du roman). La relation entre genres premiers et seconds est donc une relation de continuité, fondée sur la continuité des échanges verbaux, qui ne commencent jamais vraiment ni ne se terminent, sans qu'il soit question pour autant de progression du simple vers le complexe dans un sens comme dans l'autre. De ce point de vue, on peut soutenir que Bakhtine considère l'activité (Faïta, 2001b) à partir du texte littéraire qu'il soumet à l'analyse, et non d'un ensemble de texte, oraux ou écrits, produits dans le cadre des situations d'une sphère d'activité particulière. Il est nécessaire de prendre cette différence en considération lorsque l'on transpose à l'analyse de l'activité des notions aussi complexes que celle de genre, en ayant la prudence de considérer le nouvel objet auquel aboutit la procédure (la genèse d'une activité sur l'activitê) comme une image, ou une réplique 
déformée de la relation qui unit en sens inverse le roman à la conversation quotidienne. Il serait en effet pour le moins excessif de prétendre analyser l'activité elle-même à partir de traces écrites et/ou orales, d'images et de discours sur ces images sans prendre en compte les déformations, les diffractions qu'introduisent nécessairement les fonctionnalités et les mises en structures opérées.

C'est bien pourquoi il importe à nos yeux de considérer le résultat global de la procédure d'autoconfrontation comme une construction nouvelle, révélatrice de différences significatives entre ce qu'elle donne à voir, à comprendre, et l'activité observée, apprise et comprise au départ de l'intervention. Ce sont ces différences qui sont pertinentes, plus que les sens produits par les différents textes en eux-mêmes. On se souviendra avec profit, par exemple, de toutes les contradictions révélées par l'analyse entre l'évolution thématique du discours d'un sujet confronté et le produit de l'observation de son activité réelle. Tel conducteur de train affirmant au terme de l'autoconfrontation croisée, pour se distinguer de son collègue, “je suis de ceux qui travaillent de telle ou telle façon”, offre matière à une réflexion d'autant plus approfondie que l'observation préalable a révélé de sa part certains choix parfaitement contradictoires.

C'est à partir d'hypothèses sur le rapport dialogique que l'on peut également considérer la façon dont l'analyste, ou l'expert, joue son rôle dans la situation créée par la procédure d'autoconfrontation. Il contribue en effet à doter de certaines caractéristiques ce qui constitue pour lui une situation de travail, dans laquelle son activité propre émerge par la façon qu'il a de garantir son déroulement en fonction du genre " échanges verbaux en situation d'autoconfrontation " (composé entre autres par certaines actions verbales ayant pour fonction d'empêcher le genre de se dévoyer). Ces « micro-genres » sont cependant produits et véhiculés dans le fonctionnement du langage, dont on sait qu'il ne commence ni ne s'arrête à l'actualisation qu'en fait tel ou tel locuteur, et comme tels soumis aux lois régissant le rapport dialogique. En l'occurrence on ne considèrera aucunement l'expression de ces lois comme des règles particulières, mais au moins comme des modes de structuration acquis par l'analyste dans son appropriation des modes de déroulement discursif constituant, au fil des circonstances répétées, un " genre autoconfrontation ». Il est alors probable que le recours à un nombre indéfini de " micro-genres ", par exemple l'enchaînement question à l'autre - commentaire de ce qu'il a fait 
ou dit, sera inévitable mais variable, en fonction des ressources particulières constituées par cet analyste.

Il y a lieu de considérer également l'impact du métier premier de l'analyste sur la façon dont il gère en toute conscience sa conduite de participant à l'autoconfrontation. Qu'il soit linguiste, psychologue, philosophe, etc. ., n'est pas indifférent puisqu'il va faire appel à des ressources dialogiques spécifiques imprimant leur marque au processus auquel il participe. Ses interventions, mais aussi la façon dont il va les structurer, en fonction d'enchaînements spécifiques, puiseront dans un genre non moins spécifique proche de ce que Boutet et Fiala (1976) ont nommé une "formation discursive ». Il est clair que le rapport dialogique outrepasse largement, et dans tous les sens, un quelconque "format d'interaction » localisé au processus en cours. En d'autres termes, le guidage de l'autoconfrontation, s'il évite l'écueil de la schématisation du rapport interviewé-intervieweur, son conditionnement par un micro-genre impératif (contraintes structurelles serrées, focalisation sur les référents, rapport univoque à la tâche), ouvre en revanche la porte à l'irruption de "mondes" (François, 1989) importés par les protagonistes sans exception. Cela renforce encore, si besoin était, notre conviction que la productivité de l'autoconfrontation est entièrement liée à la rigueur avec laquelle s'organisent les conditions initiales de la mise en confrontation (voir cidessus: similarité des situations de travail présentées par les films respectifs).

Cette logique propre au processus d'autoconfrontation, que nous venons d'examiner, constitue donc le cadre dans lequel se construisent les sens concrets, c'est à dire englobant les mots et les répliques des participants en même temps que leur façon de renvoyer aux circonstances, les contextes, les relations explicites ou non dites des uns et des autres à eux-mêmes comme à cet ensemble extérieur à eux. Mais il convient encore de distinguer de cela un niveau supplémentaire : celui où se superpose un autre processus dialogique entre les chercheurs et l'objet construit, très prosaïquement le film vidéo des différentes étapes du processus d'autoconfrontation. C'est à cette occasion que se nouent un ensemble d'autres relations avec d'autres objets homologues, produits par le passé, entre lesquels (en même temps qu'avec le nouveau) on va rechercher, qu'on le veuille ou non, et sans doute identifier des constantes, des homologies . On est alors en train, en tant que chercheur, de constituer ou renforcer un genre de l'autoconfrontation, à un niveau très différent, en recherchant dans cet objet les traces des 
rapports dialogiques initiés puis développés au fil du processus, dont nous pensons qu'ils révèlent par leur mouvement ceux de l'activité. Cette phase est celle où s'engage une forme de conceptualisation et de catégorisation des caractéristiques notables de l'objet, surtout si elles paraissent récurrentes.

\section{Statut de l'objet.}

Cela repose à notre avis la question du statut de l'objet, que nous avons pressenti à un moment non comme un concentré explicite de l'activité étudiée, mais comme le déclencheur d'une réflexion ultérieure, à la fois à des niveaux différents et à un autre stade. A des niveaux différents car il a pour vocation de prendre place parmi les pièces incitant le milieu de travail récipiendaire, celui qui inclut les acteurs du processus comme les commanditaires, à prolonger le travail engagé, à remettre en question les schémas interposés entre l'activité de travail et la vision prédominante ${ }^{4}$, tout comme à proposer aux chercheurs un nouvel objet d'analyse, révélateur comme on l'a dit de liens et de contradictions significatifs avec la réalité observée.

La question se pose également à un autre stade, car ce nouvel objet joue encore de façon différente la fonction de déclencheur, en remettant en question ou en développant la réflexion méthodologique sur l'autoconfrontation, ou encore en transformant et accélérant certaines des recherches en cours en analyse du travail.

Dans tous les cas, et cette remarque s'impose si l'on considère les rôles variés que l'on peut faire jouer aujourd'hui à un tel produit (projection de films d'autoconfrontation devant des milieux de travail différents de ceux des acteurs, voire dans des classes ou autres groupes en formation), il est essentiel de bien considérer que le type d' " activité sur l'activité » initié est forcément, soumis à des lois spécifiques, tributaire de genres et microgenres différents mobilisés dans l'action de compréhension et d'appropriation.

4 Dans certains établissements scolaires " difficiles », où l'autoconfrontation a été réalisée, on a pu faire l'expérience d'une relance du processus, prise en charge par le milieu de travail lui-même, au terme de la procédure initiée par les chercheurs. On est alors parfaitement fondé à parler de processus ininterrompu (Amigues, Faïta et Saujat). 
En tant que chercheurs, il est important que nous prenions la mesure du caractère de cet objet, dont nous créons les conditions d'émergence, mais qui nous échappe et nous dépasse au terme de sa réalisation. A ce titre, on ne saurait éviter de lui attribuer un statut conforme à celui du texte, porteur de sens concrets pour ceux qui font partie de la situation de production, mais de moins en moins au fil du temps, et générateur de significations rencontrées (ou recherchées, au stade de la conceptualisation), pour ceux qui entretiennent avec l'activité initiale un rapport matériel et temporel indirect.

Au-delà du premier état du rapport établi entre le film réalisé et l'activité initiale, source des énoncés concrets supportant la mise en sens en fonction d'un genre de discours dominant et des micro-genres qui le matérialisent, il n'existe pas de frontière matérielle. Cela signifie que le chercheur peut à tout moment laisser le dialogue échapper aux lois de ce genre, voire agir lui-même dans ce sens en ouvrant des parenthèses énonciatives, initier des modalités disjointes de mise en sens. C'est le lot de tout travail d'élaboration d'un texte à partir/sur un autre texte, ce que l'on a qualifié plus haut de dialogue entre le premier niveau d'activité (activité initiale), et le second, niveau de l'autoconfrontation croisée, activité sur l'activité. La relation complexe qui s'instaure, énonciation à partir d'une autre énonciation, mise en discours dans une situation différente, hors du cadre tracé par le premier rapport, est de l'ordre du maintien de liens plus ou moins ténus, de marques persistantes du discours d'origine. On peut dire dans ce cas que le rapport dialogique initial, vidéo-discours de chaque sujet dans l'autoconfrontation primaire, n'est jamais annulé, toujours plus ou moins repérable ou sous-jacent dans la situation seconde, d'une façon assez similaire à la persistance du discours rapporté dans le discours d'un narrateur ${ }^{5}$. Bakhtine note à ce propos que dans l'énoncé du narrateur, l'énonciation primitive est intégrée à l'unité propre de la composition nouvelle, par le jeu de règles syntaxiques, stylistiques et compositionnelles. Elle conserve cependant, au moins sous une forme rudimentaire, l'autonomie primitive du « discours de l'autre » sans que celle-ci puisse être pour autant totalement appréhendée.

\footnotetext{
5 Dans l'étude des enregistrements audio d'une activité médicale en consultation clinique, les commentaires des protagonistes sur cette activité ont été repris sous la forme structurelle du discours rapporté, et ont permis de percevoir le mouvement des relations dialogiques en relation à l'activité première et l'activité seconde. Sur cette question, voir Vieira (2002a, 2002b), « Le discours rapporté dans l'activité médicale ».
} 
Il nous semble, chose essentielle, qu'en distinguant soigneusement la démarcation entre les activités successives, superposées ou enchâssées, l'analyste a la possibilité d'échapper aux lois du genre imposé par la situation seconde, et de ne pas laisser dissoudre le lien avec la situation primaire, l'activité de premier niveau. Cela le contraint sans doute à se livrer à un exercice délicat, consistant à maintenir une activité discursive autonome, intérieure, en parallèle à l'activité discursive des acteurs autoconfrontés. $\mathrm{Ne}$ pas y réussir, en revanche, fait courir le risque de voir se rompre la continuité entre les différentes phases de la procédure, rupture évoquée cidessus, et par conséquent de rester insensible à ce qui constitue le soubassement, le lien reliant l'une à l'autre les différentes phases de l'activité. L'objet autoconfrontation croisée n'est plus alors que ce qu'en font les « micro-genres » disciplinaires, les préconçus analytiques véhiculés dans la situation par des chercheurs éventuellement ignorants du danger. Le sens véritablement construit dans cette relation échappe à l'analyse.

Si l'autoconfrontation se propose d'être le processus par lequel seront captées les relations dialogiques à la frontière entre discours et activité, elle ne peut par conséquent se contenter d'en recueillir les marques offertes par les échanges verbaux. Le dialogue, pris comme activité langagière ne peut être analysé seulement en tant que somme d'énoncés produits. Il est également nécessaire d'enclencher un processus contradictoire immédiat dans lequel le choc entre le perçu et le représenté laisse émerger les rapports entre action et activité.

La pratique de l'autoconfrontation n'est pas consensuelle, et la polémique, comme genre de discours, fournit les instruments autorisant un processus qui n'est plus celui du commentaire mais du fonctionnement dialogique intérieur, orienté vers une énonciation que l'autoconfronté ne pourrait réaliser sans l'existence d'un texte matérialisé par les images et discours qui le contraignent à mettre ses paroles en concordance avec ses actes. C'est à ce moment qu'apparaissent les actions mobilisées par le protagoniste dans son aller-retour entre discours et activité, et les éléments de celles-ci. On a alors une chance de capter les thèmes représentatifs du sujet, véhiculés par son discours intérieur qui, selon Bakhtine, comporte l'expression de "toute l'essence de l'appréhension appréciative de l'énonciation d'autrui, tout ce qui peut être significatif idéologiquement..." (Bakhtine, 1929/1977: 165). 


\section{Synthèse et proposition d'un guide d'action}

Nous sommes conscients que nos réflexions révèlent à chaque reprise la nature "d'intervention clinique" de l'autoconfrontation croisée. Cela signifie que l'analyste chercheur optant pour cette démarche méthodologique devra être attentif à tout moment à sa structuration et à l'usage qu'il en fait. Il doit savoir qu'aux objectifs concrets auxquels se prête la méthode peuvent s'ajouter conjointement des effets indépendants sur les protagonistes.

L'intervention en milieu de travail ne peut être envisagée sans se défaire de la moindre illusion de neutralité, pas plus qu'il n'est possible d'ignorer les singularités des protagonistes. C'est en ce sens que nous soulignons la nature de notre objectif, dont l'ampleur est d'un niveau supérieur : il s'agit de rétablir "le pouvoir d'agir" des individus et collectifs de travail, c'est à dire faire en sorte que "d'objet d'analyse le vécu (devienne) moyen pour vivre d'autres vies" (Clot, 2001: 15).

Il s'agit aussi de rendre possible la gestion par les sujets des transformations perçues et envisagées. C'est pourquoi nous cherchons à nous entourer de garanties pour prévenir les problèmes possibles, de manière aussi à réorienter les situations au cas où ils se produiraient. Dans le cas, jamais exclu, de dommages individuels et collectifs, nous devons conserver une marge de sécurité telle qu'une reprise et un rétablissement positif soit possible.

C'est dans cette perspective que nous fournissons ci-dessous une proposition synthétique, un guide minimal d'action, avant de nous engager dans des considérations plus pratiques, accompagnées d'exemples.

L'essentiel réside selon nous dans le fait que deux types de rapport dialogique se superposent: celui initié entre les phases film, autoconfrontation 1, et par ailleurs autoconfrontation 2, et enfin dans le cadre de cette phase d'autoconfrontation « croisée ». On notera, à l'appui de ce qui précède, que cette dernière phase est le moment (l'espace-temps) d'une production discursive rendue autonome par rapport à l'activité initiale, mais incluant cette dernière comme référence. De la même façon, on peut dire que la " croisée » initie une phase de production discursive contextualisée par chacune des deux " primaires », tout en instaurant un rapport dialogique emboîtant ces contextes. On entend par là que ces 


\begin{tabular}{|c|c|c|}
\hline Phase & Nature & Caractéristiques \\
\hline Film & $\begin{array}{l}\text { Images de l'activité } \\
\text { première }\end{array}$ & $\begin{array}{l}\text { Sélection de séquences homogènes, strictement } \\
\text { comparables pour chaque participant, choisies et } \\
\text { montées en fonction de la connaissance par le } \\
\text { chercheur de l'activité et des situations de travail. } \\
\text { Première source de significations concrètes. }\end{array}$ \\
\hline $\begin{array}{l}\text { Autoconfron- } \\
\text { tation } 1^{\circ}\end{array}$ & $\begin{array}{l}\text { Production par chacun } \\
\text { des protagonistes d'un } \\
\text { discours (texte). } \\
\text { Interaction acteur- } \\
\text { chercheur. }\end{array}$ & $\begin{array}{l}\text { Discours/texte produit en référence à l'activité } \\
\text { observée. Ouverture d'un espace aux } \\
\text { commentaires du sujet, au-delà du discours } \\
\text { descriptif/explicatif et des réponses aux questions } \\
\text { du chercheur. } \\
\text { Développement de la situation. } \\
\text { Production de significations concrètes en référence } \\
\text { au film, } 2^{\text {ème }} \text { source de significations concrètes. }\end{array}$ \\
\hline $\begin{array}{l}\text { Autoconfron- } \\
\text { tation } 2^{\circ}\end{array}$ & $\begin{array}{l}\text { Production discursive } \\
\text { contextualisée (rapport à } \\
\text { la phase précédente). } \\
\text { Instauration d'un } \\
\text { rapport dialogique } \\
\text { enrichi et complexifié: } \\
\text { - Dialogue inter-activité; } \\
\text { - Rapport dialogique } \\
\text { acteur } 1 \text { - acteur } 2 \text { - } \\
\text { chercheur. }\end{array}$ & $\begin{array}{l}\text { Cette phase intègre deux niveaux de référence: à } \\
\text { l'activité initiale filmée et montée (première source } \\
\text { de significations concrètes), au contexte discursif } \\
\text { offert par le premier niveau d'autoconfrontation } \\
\left(2^{\text {ème }} \text { source). }\right. \\
\text { Ainsi, les phases d'interaction entre acteurs, } \\
\text { acteur-chercheur, Les références de chacun à lui- } \\
\text { même en dehors du processus d'interaction, } \\
\text { peuvent être rapportées aux deux sources } \\
\text { antérieures. } \\
\text { Développement de l'objet de cette nouvelle } \\
\text { activité, développement des sujets engagés dans } \\
\text { l'activité. }\end{array}$ \\
\hline $\begin{array}{l}\text { Retour au milieu } \\
\text { de travail. }\end{array}$ & $\begin{array}{l}\text { Production de l'objet } \\
\text { (résultant de l'ensemble } \\
\text { des phases) aux fins de } \\
\text { mise en patrimoine en } \\
\text { réponse à la demande } \\
\text { initiale (ou au projet). }\end{array}$ & $\begin{array}{l}\text { L'objet s'autonomise par rapport aux phases de } \\
\text { production. Il peut en être fait plusieurs usages: } \\
\text { support d'échanges consécutifs dans le milieu de } \\
\text { travail, formation, etc. }\end{array}$ \\
\hline $\begin{array}{l}\text { Appropriation } \\
\text { différée de } \\
\text { l'objet par } \\
\text { l'équipe de } \\
\text { recherche. }\end{array}$ & $\begin{array}{l}\text { Analyse spécifique de } \\
\text { l'objet produit. }\end{array}$ & $\begin{array}{l}\text { Implications conceptuelles, méthodologiques, } \\
\text { épistémologiques. L'objet lui-même, sous tous les } \\
\text { angles d'approche, comme les rapports dessinés } \\
\text { entre les stades de sa production, deviennent de } \\
\text { nouveaux objets de recherche. Le lien entre les } \\
\text { phases, les continuités préservées pendant l'action, } \\
\text { l'interface activité/discours, sont soustraits à } \\
\text { l'épreuve de la vie. }\end{array}$ \\
\hline
\end{tabular}


" primaires » offrent à la « croisée » des référents textuels, sous forme de mots, de propositions et d'énoncés, bref, d'un « déjà-là » linguistique, mais aussi -et surtout- de genres de discours sur lesquels des enchaînements à distance peuvent se produire (des manières de dire peuvent être prolongées), tout comme des enchaînements (et des oppositions) implicites sur soi, sur l'autre, sur les objets et la situation. En bref ce sont des " entours » (François, 1998) qui d'une phase, d'une situation à l'autre peuvent permettre la production de sens concrets différents par le recours à des mots et des procédés identiques dans des contextes et situations différents.

C'est précisément de l'exclusion de ces significations (et sens) concrets que l'analyste doit se préserver. Il doit pour cela, comme on l'a dit, s'appliquer à retrouver, derrière les énoncés linguistiques, les lignes rejoignant les uns aux autres les éléments présents de façon plus ou moins perceptibles dans les différentes phases. La garantie dont il dispose consiste, à ne jamais perdre de vue l'activité réelle initiale, observée par lui et filmée.

\section{Le défi proposé à l'analyste.}

Il est certains que l'analyste se voit toujours plus ou moins confronté à une sorte d'épreuve. Il doit se situer dans l'interaction avec les participants, se trouvant dans une position énonciative particulière à chaque étape de la procédure, sans perdre de vue les dimensions concrètes de l'échange en maintenant un rapport permanent avec l'activité initiale et les éléments de contextualisation (voir ci-dessus). Le maintien de cet équilibre est déterminant, car c'est de lui que dépend la productivité réelle du processus, à travers les différentes situations créées. Que sa posture énonciative se borne à celle d'un participant à l'interaction située dans l'autoconfrontation croisée, et la porte sera ouverte en grand aux « micro-genres » des spécialités évoqués plus haut... Il est donc de la plus haute importance, on y insiste, qu'il se situe consciemment en un point de la continuité générale entre phases de l'action, tout en conservant une vision réaliste des lacunes de son point de vue, de façon à y pallier dans toute la mesure du possible ${ }^{6}$.

\footnotetext{
6 La meilleure des volontés ne peut annuler le fait que chercheurs et acteurs n'appartiennent jamais à la même histoire.
} 


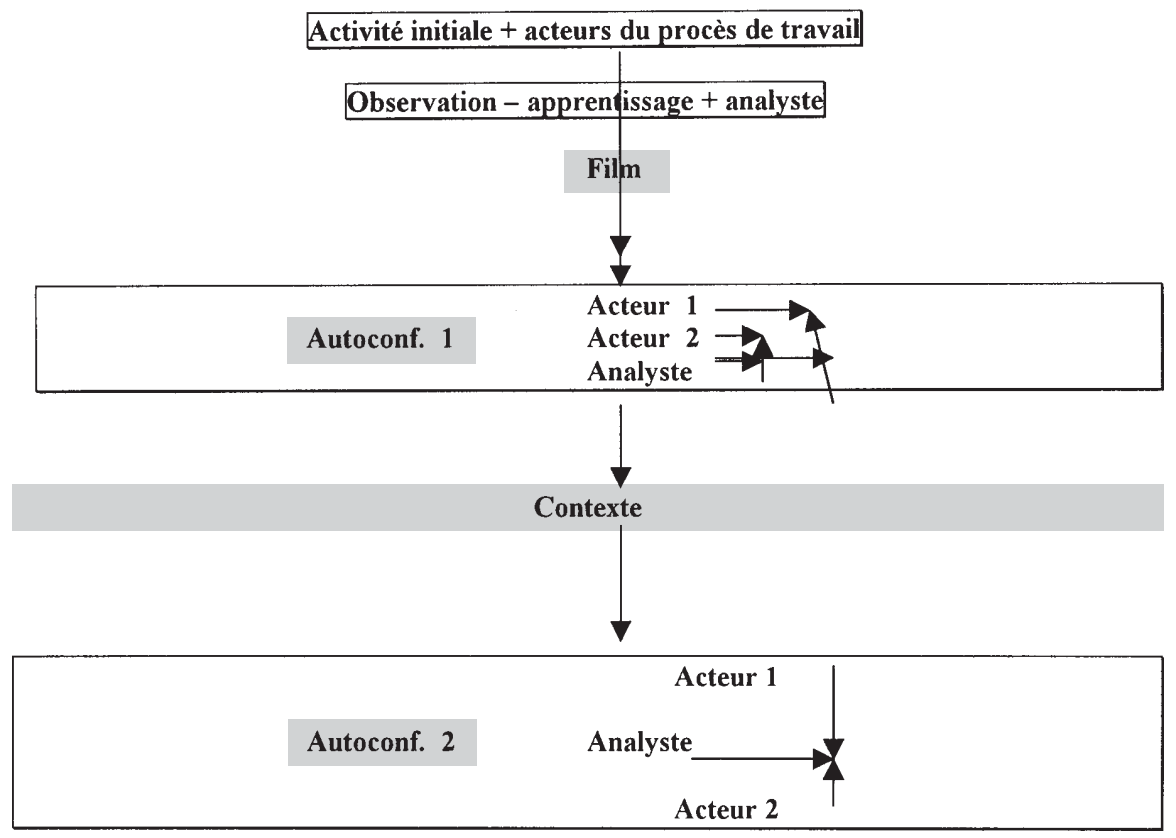

On figurera grossièrement comme suit les rapports respectifs entre les protagonistes du processus, et les situations respectives dans lesquelles ils se trouvent au gré des phases successives.

Pour simpliste qu'il fût, le schéma fait apparaître les barrières et les continuités déjà signalées. L'analyste, du fait qu'il n'appartient pas, ou partiellement, à la sphère d'activité concernée se situe en position inégale par rapport aux autres protagonistes. Il devra donc compenser, modaliser, les conséquences de cette inégalité objective en se livrant à un double travail au niveau de l'activité d'échange langagier.

Il devra procéder à des aller-retours entre les significations produites dans le cadre des discours respectifs des partenaires interagissant entre eux et avec lui, et le résultat des ses propres observations, retravaillées dans la phase d'autoconfrontation simple. C'est à ce prix qu'il pourra s'engager dans le nécessaire va-et-vient entre "l'horizon immédiat " (Bakhtine, 1929/1977: 150) du groupe auquel il est momentanément associé, avec les deux acteurs de l'autoconfrontation croisée, et « l'horizon social élargi » du groupe social non-restreint auquel appartiennent ses interlocuteurs. Pour ceux-ci en effet, l'espace d'interaction défini par la procédure et la 
situation créée ne recouvre pas, et de loin, cet autre espace englobant de leur activité, fait d'un univers de pensée, de cultures de métier, de genres d'activité et de genres professionnels (Clot et Faïta, 2001). Or c'est dans ce dernier, qui n'est pas immédiatement accessible à l'analyste, que sont stratifiées les composantes du rapport dialogique entretenu par les acteurs. Il n'est pas réaliste d'imaginer que le décalage en question puisse pour autant être comblé mais, et c'est la nature du défi proposé à l'analyste, il devra tenter de se rapprocher au maximum des modalités de l'échange fonctionnant dans le cadre de cet " horizon » élargi dont il est objectivement exclu. Pour cela, sa principale ressource consistera à utiliser la continuité entre les phases successives pour construire un troisième type d'espace, proche de cet espace-temps déjà mentionné, fonctionnant comme lieu de conjonction des activités respectives des protagonistes, ou encore comme « territoire commun » des uns et des autres où les mots produiront à la fois sens concret et signification. Ce territoire ne se réduira pas à l'exiguïté de l'interaction en situation d'autoconfrontation, mais sera relié par le plus grand nombre possible de " ponts » aux autres domaines d'activité concernés, ce qui est le propre des échanges évoluant dans le cadre d'un rapport dialogique réel. La notion de thème, propre à Bakhtine, inséparable d'une conception élargie de la signification, convient parfaitement pour décrire le travail à accomplir, et les conditions (cidessous : conditions d'une énonciation concrète ») à créer pour le faire :

“... le thème constitue le degré supérieur réel de la capacité de signifier linguistique. (...) la signification ne veut rien dire en elle-même, elle n'est qu'un potentiel, une possibilité de signifier à l'intérieur d'un thème concret. La recherche de la signification de tel ou tel élément linguistique peut (...) aller (...) vers le degré supérieur, le thème : dans ce cas il s'agira de la recherche de la signification contextuelle d'un mot donné dans les conditions d'une énonciation concrète". (Bakhtine, 1929/1977: 145.)

On empruntera l'exemple qui suit à une étude conduite en clinique de l'activité':

Au cours de cette étude consacrée au " stress » des contrôleurs SNCF, une jeune femme, SW, participe à la procédure d'autoconfrontation. Au cours de la phase 1, elle procède au commentaire et à l'explicitation de la situation de travail représentée

\footnotetext{
7 Gabriel Fernandez, Frédéric Yvon, « Enquête sur le stress professionnel », Clinique de l'Activité, Laboratoire de Psychologie du Travail, Paris, CNAM, Novembre 2001.
} 
par le film. Pour rendre comprébensibles par les chercheurs certains des gestes et des postures que l'on voit à l'écran, elle évoque les dangers liés à certains défauts du type de wagons utilisés, notamment le fait qu'un voyageur inconscient peut ouvrir les portes en cours de route.

SW. .. dans ce type de matériel les portes restent ouvertes entre les gares...c'est vrai qu'un adulte conscient va pas s'amuser à ouvrir les portes et à sauter.. mais un gamin c'est tout à fait possible..

(...) une maman qui fait pas attention.. le gamin il ouvre les portes et il tombe... on a tout gagné !! (...).

GF (chercheur) ... on a tout gagné ? C'est l'enfant qui ouvre qui a tout gagné ?

SW c'est à dire.. un enfant qui ouvre.. il tombe dessous.. on a un mort sur la conscience après..

GF Qu'est-ce que vous avez gagné ?

SW ... c'est une expression.. quand je dis « on a tout gagné »... c'est l'entreprise qui a tout gagné.. qui ne fait rien contre çà.. la direction est consciente que ces rames sont dangereuses et çà fait quand même quelques années qu'elles roulent !!

Dans cet échange le chercheur relève une expression familière fonctionnant par anti-phrase: "on a tout gagné" signifie au contraire qu'une catastrophe est survenue. Il serait possible de s'en tenir, en l'occurrence, à la signification, ce que ne fait pas le chercheur. Celui-ci réagit en fonction d'un doute nourri par sa culture professionnelle, puisqu'il connaît l'entreprise de l'intérieur. C'est ce qui explique le refus, de sa part, d'en rester au niveau de la signification abstraite (“c'est l'enfant qui...") et motive la seconde question.

Cette attitude permet à l'énoncé concret d'émerger là où peut-être il serait demeuré dans le non dit: les contrôleurs travaillent dans des conditions rendues plus précaires que la normale par l'attitude de la direction. Aux accidents possibles pour des raisons objectives s'ajoutent ceux, prévisibles, qui peuvent survenir en raison d'une politique délibérée.

Une position similaire pourrait parfaitement être tenue par un chercheur moins familier de la SNCF mais observateur attentif, soucieux de mettre en relation les actes discursifs non seulement avec les éléments de la situation, mais aussi avec ce qu'il perçoit de "l'horizon élargi" de ses interlocuteurs. 


\section{Peut-on modéliser la conduite de l'autoconfrontation ?}

L'originalité de l'autoconfrontation, en tant que méthode de sollicitation de l'expérience et des savoirs en actes, réside dans la libération des façons de signifier offertes au sujets, libération permise par le déploiement d'un rapport dialogique nouveau, échappant aux contraintes des situations vécues antérieurement. Ainsi, la pluralité des mises en relation possibles entre les énoncés et les situations et actions de référence peut devenir elle-même objet de réflexion et de débat, à travers la reconnaissance de la pluralité des voix, la pluralité des signes, qui composent la dimension concrète des échanges verbaux.

A la différence des méthodes de conduite d'entretien ${ }^{8}$, l'autoconfrontation ne saurait être guidée en privilégiant une activité du sujet centrée sur la seule référence au vécu, et en excluant par conséquent toute forme de digression ou de modalisation. L'exemple cité ci-dessus montre au contraire quel rôle important, pour la connaissance de l'activité au sens large, peut jouer la digression métadiscursive.

La question des modes et formes de la relance du processus dialogique ne s'y pose donc pas de la même façon. Puisqu'il ne s'agit pas d'empêcher toute dérive du discours par rapport à une "position de parole incarnée" du sujet (Vermersch), il n'existe pas en propre d'actes de relance spécifiquement appropriés, par exemple des formes de questions « ancrant » le sujet dans la réalité évoquée.

\section{Construire une situation favorisant le dialogue.}

Si l'on se place en effet d'un point de vue privilégiant le rapport dialogique comme moteur du processus d'élaboration et d'expression des savoirs, de l'expérience des sujets, on doit alors admettre que cette expérience participe de l'activité, car elle est présente, en tant que facteur direct ou indirect des choix effectués dans l'action. Mais rien n'autorise à considérer a priori que la part de cette expérience susceptible d'être

8 On ne s'attardera pas sur l'évocation de ces méthodes, en se contentant de signaler les travaux de P.Vermersch sur " l'entretien d'explicitation », utilisée en matière se sollicitation de l'expérience professionnelle. 
formalisée, structurée et mise en mots, puisse être légitimement privilégiée par l'investigation, en d'autres termes qu'elle en constitue la seule part à faire émerger et à retenir. Non seulement, en effet, la part directement exprimable de l'expérience n'épuise pas tout à elle seule (il suffit de penser à tout ce qui, dans l'ordre de l'émotion et des affects, joue un rôle important sans pour autant trouver à se dire au moyen de ressources strictement linguistiques), mais encore la mise en mots constitue beaucoup moins une médiation entre le vécu et sa connaissance que certains moments d'une activité où la part du développement, du déclenchement de mouvements nouveaux transformant des relations que les protagonistes croyaient stabilisées. Or, comment préjuger des voies et des formes de ce mouvement dialogique, de la nature des thèmes trouvant à y circuler, des genres de discours choisis pour le supporter ?

Dans l'exemple suivant, extrait d'une autoconfrontation croisée (la première du genre ${ }^{9}$ ), les chercheurs présents n'interviennent pas, et la "motricité du dialogue" joue à plein.

Autoconfrontation croisée. Les deux AdC TGV ont assisté à l'autoconfrontation $n^{\circ} 1$ de chacun. Ils ont entendu leurs commentaires respectifs, et ont constaté des différences sensibles dans leurs façons de faire. L'échange évolue sur certaines de ces différences, visibles sur les films

L'intérêt majeur de passage, analysé par ailleurs (Faïta, 2001a), réside dans le fait que le dialogue évolue à partir du thème de la vitesse pour gagner celui de la qualité du service, puis celui du soin du matériel. Cette cascade, de thème en sous-thèmes successifs, montre bien que des valeurs importantes peuvent exister en arrière-plan, dissimulées par des mots anodins. Il est évident, par ailleurs, que les mimiques jouent dans ce fragment un rôle non négligeable, notamment au niveau des enchaînements d'un interlocuteur sur l'autre. L'intervention d'un membre de l'équipe de recherche, décidé à réguler et recentrer le débat, aurait pu entraver ce développement.

Pour avoir à de multiples reprises procédé à la sollicitation de l'expérience professionnelle et des savoirs en actes de travailleurs de tous

9 Il s'agit d'une situation réunissant deux conducteurs de trains, à l'occasion d'une recherche réalisée en 1996 par Faïta, Dagand \& Pelegrin au dépôt TGV de Lyon-Mouche (Faïta, Clot, et al., 1996). 


\begin{abstract}
AdC1
Moi je roule beaucoup.. je fais partie de ceux qui.. c'est limité à 155 je roule à 155 .. pas à 145 !.. disons que je travaille, disons.. par paliers (..) s'il faut être à 141 au PK 21.. je commence à freiner au PK 22.. je ne m'y prends pas à l'avance.. la vitesse c'est la vitesse. . j'aime bien travailler à la vitesse maxi et rouler par paliers..
\end{abstract}

Moi çà ne me dérange pas d'arriver en avance.. c'est pour çà que je travaille par paliers. je flirte avec la vitesse maxi.. avec les limites

Parce que moi arriver en avance çà ne me dérange pas.. quand les conditions s'y prêtent bien çà ne me dérange pas du tout d'arriver avec $5 \mathrm{mn}$ d'avance.. çà ne dérange personne dans le service.. au contraire çà ne peut qu'arranger le service..

Expression de perplexité

Moi j'ai pas l'impression de brusquer le matériel.. mais c'est vrai que je le sollicite au maxi.. je profite au maximum de tout ce qu'on m'offre...
Pendant le tour de parole de son collègue, la mimique de $\mathrm{l}^{\prime} \mathrm{AdC} \mathrm{n}^{\circ} 2$ traduit un étonnement croissant

\section{AdC2}

Alors là.. je fais tout le contraire moi.. sous réserve bien entendu d'être à l'heure.. je laisse chuter naturellement la vitesse..

Expression de stupéfaction

Ah bon ! ! !
.. moi arriver en avance je fais pas trop.. je préfere ménager le matériel.. des fois çà casse...

ordres, nous avons aussi pu constater comment la catégorisation dans la mise en mots ou la mise en discours ne leur offrait qu'un registre parmi d'autres possibles. L'échange verbal offre en effet de multiples ressources potentielles au développement de l'activité dont il participe. Parmi cellesci, tous les procédés empruntés à la gamme des rhétoriques - de la plus formelle, avec ses métaphores, ses catachrèses ou litotes, à la plus coutumière, univers des comparaisons familières, des accentuations par répétitions, ou par énumération, etc., fréquentes dans le discours du travail (Faïta, 1989) - mais aussi de la variation prosodique, du jeu dramatique...

L'échange verbal, domaine du rapport dialogique, offre à celui-ci une gamme sur laquelle jouer, en usant au maximum de la plasticité du signe linguistique (il n'y a jamais une relation possible et une seule entre les éléments de la réalité et des mises en mots correspondantes), et de sa mise 
en contraste avec la multitude des autres signes : la communication humaine ne relève pas d'une sémiologie unique.

Privilégier un canal, la mise en mots encadrée par un appareil de règles, réduite au jeu des strictes références et inférences sémantiques c'est faire la part excessivement belle à des mécanismes édulcorant l'histoire des sujets. C'est en outre se contenter d'un cas particulier, voire d'une illusion de communication, fondée sur l'hypothèse (on est tenté de dire le pari) qu'il existe des expériences réelles, constituées dans une multitude de situations concrètes, et trouvant leur contre-partie intégrale dans une production discursive conforme au code, ses unités et ses règles.

\section{"Micro-genres" contre "circulation".}

La méthode privilégiant l'encadrement de l'expression des opérateurs au moyen de techniques appropriées, ou, aussi bien, le guidage traditionnel des entretiens, peut avoir pour effet de conditionner la parole par diverses formes de schématisation imposées, inconvénient que l'on souhaite précisément éviter au moyen de l'autoconfrontation croisée.

Il est impossible en effet d'éviter les conséquences du jeu des questions et des réponses, ou tout simplement de l'asymétrie imposée au dialogue par une conduite unilatérale de celui-ci. Le rôle de celui qui questionne, ou qui, de quelque façon que ce soit, monopolise l'initiative des enchaînements entre les thèmes abordés, entrave les échanges de places entre interlocuteurs, phénomène bien connu de l'analyse des interactions.

Suivant les termes déjà utilisés, ce processus a toutes chances d'aboutir à l'apparition de "micro-genres", c'est à dire à des rapports, structures et formes préexistant aux énoncés produits par les sujets et s'imposant à eux dans une large mesure (cf. ci-dessus) ou, tout au moins, contraignant le sujet parlant à se définir par rapport à eux : rompre un enchaînement question/réponse est toujours possible, mais produit alors une véritable signification. Celui qui est en mesure de questionner l'autre systématiquement se positionne dans un rapport de domination. Le trait est renforcé lorsque la question est en fait instrumentalisée, dans le but d'imposer à l'interlocuteur une orientation et des choix expressifs et argumentatifs auxquels il n'aurait pas procédé de lui-même.. Dans ce cas, 
c'est le rapport, illustré et maintenu par une certaine variété de procédés, qui fait sens, et non les énoncés par eux-mêmes.

Sur un autre terrain, pas si lointain, Amigues fait remarquer que le "genre scolaire", donne forme à l'apprentissage (Amigues et ZerbatoPoudou ). Les façons de conduire la classe "ritualisent" en effet les rapports au sein du groupe, entre le groupe et ses interlocuteurs. Malgré la brièveté (d'ailleurs relative) des rapports de questionnement ou d'interview, on voit bien comment ceux-ci peuvent produire un type d'assujettissement aux règles d'un rituel implicite chez des sujets renvoyés à la seule dimension référentielle de leur propos, à l'exclusion de toute forme de digression, d'évaluation métacognitive ou métadiscursive.

Il est assez clair que de telles modalités de conduite de l'expression entrent en contradiction formelle avec l'idée d'une démarche ouverte sur la production discursive conçue comme instance de développement, surtout, des capacités du sujet : capacités à mettre à profit le rappel de son vécu, sa confrontation à autrui et à l'image de soi que lui retourne l'autre, la mise en circulation des idées supportées par les thèmes du discours, le mouvement propre de celui-ci dans le cadre de l'activité d'échange. Toutes sollicitations pour réélaborer ses points de vue sur les objets de son activité et sur luimême comme acteur.

Le mouvement imprimé par ces différentes confrontations s'ajoute à ce que nous qualifions de "motricité du dialogue" (Clot et Faïta, 2001) c'est à dire à cette propriété du rapport dialogique conduisant les opérateurs sur la voie de la transgression du déjà dit, du "précodé" ou du donné, pour s'engager dans la création, et devenir au minimum leur propre interlocuteur.

\section{Pour quelles raisons privilégier le rapport dialogique et le sens concret?}

L'hypothèse méthodologique fondatrice de l'autoconfrontation croisée accorde une place prépondérante au rapport dialogique, et non au seul dialogue, entendu comme enchaînement et succession de répliques formelles. Les "sphères" d'activité qu'évoque Bakhtine, c'est à dire toutes les façons de recourir au langage pour accomplir une tâche, atteindre un but, gérer une relation englobent l'ensemble des dimensions temporelles de l'activité (passé, présent et futur), mais aussi l'irréel et l'hypothétique, 
puisque le locuteur adresse simultanément son énoncé à l'autre, l'interlocuteur présent, aussi bien que le "surdestinataire" idéal, celui qui interprètera à la perfection les éléments matérialisant son projet discursif, son "vouloir-dire" (Bakhtine, 1984).

Il est donc évident que le dispositif à mettre en place pour favoriser l'engagement de ce processus ne saurait reposer sur des conceptions limitatives visant-ou ayant pour effet- de brimer en tout ou partie la dramatique" de production du sens qui, dans chaque énoncé, se joue sur les trois plans à la fois. Ni la relation au contexte, ni les rapports référentiels, ni les inférences sémantiques constatées en situation d'interaction ne peuvent permettre à eux seuls de rendre compte de ce phénomène. La signification, abstraite et prévisible, produite par une phrase (ou une proposition, au sens de Bakhtine), qui pour être complète sur le plan syntactico-sémantique n'en est pas moins une figure unidimensionnelle représentant superficiellement l'énoncé, dont la part concrète ne peut trouver à se manifester hors d'un développement faisant appel de façon variable à la dimension historique du processus, au contexte, ou encore à une phase ultérieure de ce développement.

Dans l'exemple suivant (Amigues, Faïta \& Saujat, 2002) l'autoconfrontation met en présence deux professeurs exerçant dans des classes « difficiles ». Après avoir visionné l'autoconfrontation 1 de l'un d'entre eux, le dialogue évolue sur le thème de l'arrivée des élèves retardataires. Ceux-ci dérangent-ils ou non leurs camarades déjà au travail?

B. .. et les élèves sérieux qu'on voit à l'image là, ils sont pas dérangés par l'arrivée tardive de ces deux élèves ? ... ils te demandent pas .. heu... « virezles " parce que nous on est déjà en activité.. ?

C. .. non ! ... j'ai pas de remarques, parce que eux, en fait ils sont en activité, ils arrivent .. ils ont un truc à faire.. ils savent faire en plus parce que.. le premier exercice que j'ai donné, hein, c'est le dernier qui a été fait le cours d'avant... donc ils arrivent... théoriquement ils savent faire... donc eux ils sont occupés.. (...) çà permet justement de gérer l'arrivée des élèves... en vrac..

Dans ce cas la contradiction évidente sur le segment d'énoncé « sont en activité » illustre parfaitement l'intérêt de la méthode, la nature de son apport. Celui-ci n'est pas direct, la part d'information objectivement délivrée 
n'a pas d'importance en soi, mais ce fragment est une pièce parmi d'autre dans un mouvement, une circulation et une confrontation des thèmes qui révèle aux acteurs les contradictions sous-jacentes à la façon dont ils expriment leurs savoirs en actes, d'une façon qu'eux-mêmes ne soupçonnaient pas. Dans ce cas précis, la divergence sur la nature de « l'activité » recouvre en fait un conflit thématique ( occuper » les élèves c'est non seulement les mettre formellement au travail mais surtout leur permettre de « savoir » faire). Celui-ci va évoluer par la suite, la radicalisation des posture des deux professeurs va évoluer vers une phase de mésentente, de rupture de l'interaction, avant de se conclure par une évolution du premier nommé vers les positions de son collègue.

Le chercheur, qui s'est manifesté avant ce passage, ne doit surtout pas intervenir à ce moment là. Une question de sa part, ou un soulignement de la contradiction, aurait certainement pour effet de bloquer le développement en cours.

Le sujet se trouve en définitive confronté à cette contradiction aussi permanente que systématiquement renouvelée : exprimer des relations infiniment complexes et variables au moyen d'outils rudimentaires, en petit nombre, entrant dans des combinaisons ayant déjà fait leur office dans des circonstances dépassées.

Il ne lui reste donc comme ressource que de découvrir, plus ou moins par lui-même, plus ou moins avec l'aide des autres, plus ou moins contre eux, des façons « ouvertes » de signifier, en introduisant de la variété dans la façon d'utiliser un matériel verbal déjà existant et a priori impropre à un usage singulier. Il n'est donc pas étonnant qu'il découvre, la plupart du temps, les ressources nécessaires à son expression au fil de son implication dans l'interaction, dans des situations de confrontation où son activité se heurte à celle des autres ou s'y mêle. En même temps, et bien qu'il s'agisse là encore, d'une affaire de proportions, il ne suffit pas de dire que l'interaction, la référence contextuelle des actes de langage suffisent à valider les productions discursives de ce sujet car, comme on l'a vu, le processus dans lequel est engagé celui-ci déborde, et de loin, les limites de la seule situation. 


\section{Garantir la productivité dialogique en préservant la production du sens concret.}

L'interrogation récurrente sur la nature et la forme des « questions à poser » lorsque l'on guide une autoconfrontation, simple ou croisée, n'est pas dépourvue de pertinence, puisque l'on se trouve effectivement, observateur/chercheur/enquêteur et acteur/sujet, impliqués dans une situation créée à des fins précises : avancer dans la connaissance de l'activité par l'engagement dans une autre forme d'activité.

Il importe donc de prendre la mesure de ce qui sépare de telles situations à la fois de celles de la vie courante, fussent-elles exceptionnelles, et de celles que génèrent habituellement les pratiques d'entretiens. Le rapport dialogique instauré dans l'autoconfrontation présente ceci de particulier qu'il inclut un tiers étranger à l'histoire propre du sujet autoconfronté, sans que le croisement des activités respectives de l'un et de l'autre ressortisse - ou du moins pas à l'identique - à l'inégalité caractéristique du rapport enquêteur enquêté.

- La question est alors de savoir si cet étranger» peut n'être pas seulement l'interviewer, celui qui dans l'entretien ordinaire ou d'explicitation s'efforce de maintenir les énoncés produits dans un rapport exclusif de contextualisation avec l'entourage qu' ils constituent eux-mêmes, les uns pour les autres, mais une source d'optimisation des mécanismes à l'œuvre dans la production du sens par le rapport dialogique : anticipation maximale du travail de compréhension d'autrui, efficacité des différents modes de catégorisation intervenant dans la mise en mots (lexicale, syntaxique, stylistique...), dynamique interactionnelle, etc.

La difficulté qui se révèle au fur et à mesure que l'on progresse dans le sens d'une définition du rôle, c'est à dire très clairement de ce que doit contribuer à créer, en matière de rapports inédits, l'observateur participant à la situation d'autoconfrontation, tient à ce que l'objectif n'est pas unilatéralement défini, et ne saurait l'être en dehors des rapports construits avec le ou les partenaires concernés. Le sens de l'activité ne se résume pas à la sollicitation ou à "l'extraction » de données, dans le cadre d'une procédure « empirique ». Il est à rechercher dans la relation qu'institue la procédure entre les activités conjointes des participants et la construction concertée d'un objet nouveau, à soumettre au travail d'investigation des 
uns et des autres. C'est bien pourquoi la focalisation sur l'expression, ou la mise en mots de relations bi-univoques entre éléments de discours et éléments d'expérience est invalide dans ces circonstances. C'est d'un déplacement qu'il s'agit, avec toute la productivité que l'on est en droit d'en espérer : le développement des situations revisitées, la démultiplication des explications et hypothèses favorisées par un processus créant les conditions d'une libération des "façons de signifier ».

Le sujet soumis à l'autoconfrontation, d'abord simple, doit se trouver dans des conditions lui permettant de s'ouvrir à lui-même ces possibilités, en trouvant les ressources nécessaires dans l'écart entre ce qu'il constate de lui-même en action, de comment cette action se dit et communique aux autres, et par ailleurs ce qu'il éprouve le besoin de recréer comme significations, d'abord pour lui-même, en réaction à ce constat.

Il s'agit là de ce rapport " d'étonnement » à soi et sur soi (Clot, Faïta et al.), dont la communication exige l'ouverture à la dimension concrète des façons de signifier.

Il s'agit en premier lieu de rompre avec la pure logique argumentative, avec ce que l'on a qualifié de " rhétorique savante ou coutumière » celles qu'installent précisément les déclinaisons successives des genres et sousgenres (on a utilisé ci-dessus « micro »), et qui contiennent l'expression dans la référence massive au donné, au " déjà-là », parfois même jusque dans la façon d'innover... La rupture consistera à ouvrir les portes, au contraire, aux autre substrats de la communication, principalement aux rapports contradictoires, généralement brimés, entre ce que les sujets perçoivent à la fois comme devant être dit et comment le dire, et ce qu'ils pressentent qu'ils pourraient dire d'autre en faisant sens autrement.

\section{Pour conclure.}

Proposer un « modèle » en matière de conduite de la procédure d'autoconfrontation serait sans aucun doute en parfaite contradiction avec les fondements de la démarche, et d'abord avec le fait qu'aucun processus, quand bien même il serait consécutif à la création d'une situation ad hoc, ce qui est le cas, ne peut se voir assigner un objectif univoque. Créer les conditions d'un rapport dialogique entre deux interlocuteurs c'est créer les 
conditions pour que le dialogue entre eux cherche ses bases, puis évolue et transforme ces mêmes bases au fil du mouvement. Le fait que les initiateurs puissent attendre du rapport noué qu'il évolue dans un sens plutôt que dans un autre, que les positions des interlocuteurs se transforment d'une manière conforme à l'intérêt de l'enquête, n'est pas contradictoire. Mais, comme on l'a amplement montré au début, c'est en amont, dans le cadre des précautions à prendre, des choix à faire, dans la définition stricte de la place et de l'attitude de l'analyste qu'il faut situer les problèmes et leur solution.

Ce dernier doit en revanche se positionner, au cours du processus, de manière à ne pas biaiser les rapports par des manifestations qui pourraient introduire, bon gré mal gré, des sous-genres discursifs et comportementaux que ses partenaires ne pourraient manquer d'identifier ou ressentir comme autant de tentatives pour infléchir leur propre conduite ver un objectif opaque. Souvenons nous du fait qu'il n'existe aucune neutralité bien pensée en matière d'échange verbal. Les mots de l'un " procèdent de lui autant qu'ils sont dirigés vers l'autre »(Bakhtine). Il faudrait donc que l'analyste détienne une vision claire du devenir du dialogue, en rapport avec des objectifs eux-mêmes limpides, pour jouer en toute impartialité un rôle d'omniscient - omniprévoyant.

Rappelons (voir ci-dessus, en première partie), que pour nous le chercheur doit éviter de confondre son activité avec celle des participants à l'autoconfrontation croisée. Il doit se centrer sur l'activité concrète des interlocuteurs, accéder dans la mesure du possible à la dimension concrète de leurs énonciations, et non se concentrer sur la partie hic et nunc de leurs conduites en situation d'interaction. Il ne s'agit pas, en effet, de créer une nouvelle référence pour les énoncés produits dans ce cadre, ce qui se retournerait contre les principes affichés.

L'essentiel demeure de respecter la continuité réunissant l'activité initiale, vécu des uns, produit de l'observations des autres, à la phase croisée, en cheminant par les étapes de la mise en image et de la première mise en mots des situations de travail dans l'autoconfrontation simple. Rappelons, avec la plus extrême prudence, justifiée par les risques inhérents au transfert d'une théorie élaborée pour d'autres objets, ce qu'écrivait Bakhtine : « .. ce n'est pas dans le contexte des valeurs de ma propre vie que mon vécu peut acquérir sa propre signification (...). Dans ma vie ce contexte n'existe 
pas pour moi. Il me faut un point de fixation du sens qui soit situé hors du contexte de ma vie, qui soit vivant et créateur »(Bakhtine, 1984, p.123).

Ce point de fixation du sens, nous essayons de le créer en ouvrant aux sujets une " parenthèse » où ils peuvent devenir « autre par rapport à eux-mêmes »(Bakhtine, ibidem), tout en éclairant le processus par lequel évolue la transformation. Dans l'autoconfrontation croisée, c'est le pair, l'alter ego qui fournit ce point de fixation, à partir duquel le sujet peut alors se saisir de son propre vécu en rapport avec la situation de travail et l'objet de ce travail.

E-mail: mvieira@hotmail.com Recebido em outubro de 2002 Aprovado em março de 2003

\section{RÉfÉrence Bibliographique}

Amigues, Renée, Daniel Faïta et F. Saujat. 2002. L'autoconfrontation: un mode d'investigation fondé sur la co-élaboration des savoirs professionnels et le développement des compétences. In: Communication au Colloque "Politique des Savoirs", 28-29 Juin 2001, Université Lyon 2.

Amiques, R. \& T. M. Zerbato-Poudou. 2000. Comment l'enfant devient élève. Paris: Retz.

BakHtine, M (Volochinov, V. N.) 1929/1977. Le marxisme et la philosophie du langage. Paris: Minuit.

. 1929/1992. Marxismo e filosofia da linguagem. 6 a edição. São Pau-

lo: Hucitec.

. 1975-1993. Esthétique et théorie du roman. Paris: Gallimard.

. 1929/1961-1962/1970. La poétique de Dostö̈evski. Traduction d'Isabelle Kolitcheff. Preface de Julia Kristeva. Paris: Éditions du Seuil.

Boutet, Josiane, P. Fiala, e J. Simonin-Grumbach. 1976. Sociolinguistique ou sociologie du langage. Critique, 344: 68-85.

Clot, Yves. 2001. Clinique de l'activité et pouvoir d'agir. Education Permanente, 146: 7-17.

Clot, Yves. \& Daniel Faïta. 2000. Genre et style en analyse du travail, concepts et méthodes. Travailler, 4: 7-42. 
Clot, Y; Daniel Faïta, Gabriel Fernandez e Livia Scheller. 2001. Entretiens en autoconfrontation croisée: une méthode en clinique de l'activité. Education Permanente. Dossie: Clinique de l'activité et pouvoir d'agir. Genève, n. 146/2001-1:17-25.

FAÏTA, Daniel. 1989. Mondes du travail et pratiques langagières. Langages, 93: 110-124.

. 1997. La conduite du TGV: exercises de styles. Camps visuels, 6: 75-86.

. 2001a. Genres d'activité et styles de conduite. In: Borzerx, A. et Fraenkel, B. Langage et Travail, Communication, cognition, action. Paris: CNRD Editions: 264-282. . 2001b. L'analyse du travail et le statut de l'activité chez Bakhtine. Travailler, Revue Internationale de Psychopathologie du Travail, 6: 13-30.

FAїтA, Daniel \& Yves Clot (dirs.) 1996. Signer la ligne. In: Recherche sur la dimension bumaine de la conduite des trains, SNCF - CCE-SNCF. Marseille, IREPT.

FrançOIs, Frederique. 1989. De quelques aspects du dialogue psychiatrepatient. Places, genres, mondes et compagnie. CALaP, n. 5: 39-92. . 1993. Pratiques de l'oral. Paris: Nathan. . 1998. Le discours et ses entours. Paris: L'Harmattan.

Todorov, Tzvetan. 1981. Mikhaïl Bakbtine le Principe Dialogique suivi de Écrits du Cercle de Bakhtine. Paris: éditions du Seuil.

Vermersch, P. 1994. L'entretien d'explicitation. ESF.

Vieira, Marcos. 2001. Le role du dialogisme dans l'analyse de l'activité de travail. In: IIIèmes Rencontres APST/APRIT. Marseille, Université de Provence/Centre Saint-Charles. 5 p.

. 2002a. O discurso relatado na atividade médica. In B. Brait, B \& C. Souza e SiLva (orgs.) Discursos: Teorias e Práticas. São Paulo: EDUC, no prelo.

. 2002b. A atividade, o discurso e a clínica: uma análise dialógica do trabalho médico (Tese de doutorado em Lingüística Aplicada e Estudos da Linguagem) PEPG LAEL PUC-SP, São Paulo. 